\title{
Lumpy Skin Disease in Armenia
}

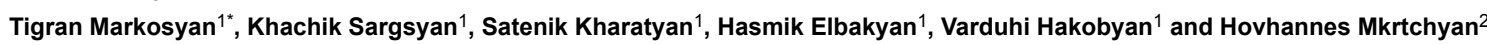

${ }^{1}$ Scientific Centre for Risk Assessment and Analysis in Food Safety Area, SNCO, SSFS, MOA of the Republic of Armenia, Yerevan, Armenia

${ }^{2}$ State Food Safety Service of the Ministry of Agriculture of the Republic of Armenia, Yerevan, Armenia

"Corresponding author: Tigran Markosyan, Scientific Centre for Risk Assessment and Analysis in Food Safety Area, SNCO, SSFS, MOA of the Republic of Armenia, Yerevan, Armenia, Tel: +374 930005 63; E-mail: tigran79hm@yandex.ru

Rec date: October 14, 2017; Acc date: November 02, 2017; Pub date: November 03, 2017

Copyright: (c) 2017 Markosyan T, et al. This is an open-access article distributed under the terms of the Creative Commons Attribution License, which permits unrestricted use, distribution, and reproduction in any medium, provided the original author and source are credited.

\begin{abstract}
Lumpy skin disease is a poxvirus disease of economic importance. The first clinical cases of LSD in Armenia were registered in December 2015 among cattle which further was confirmed in laboratory in local and international levels. The source of LSDV was not identified and the role of vectors is not clear but there are some insects presented in country and which are known as a vector of LSDV transmission. For the control and prevention of the further spread of LSDV the vaccination with sheep pox live vaccine was conducted. During 2016 and first 9 months of 2017 in total 487518 cattle were vaccinated. In 2016 about $50 \%$ of total cattle population, which were located in risk zones, were vaccinated and in parallel with other control measures it have helped to stop further spread of LSDV in country.
\end{abstract}

Keywords: Lumpy skin disease; Lymph nodes

\section{Introduction}

Lumpy skin disease (LSD, knopvelsiekte) is a pox disease of cattle caused by virus which is classified in the genus Capripoxvirus within the subfamily Chordopoxvirinae of the family Poxviridae [1] and characterized by fever, nodules on the skin, mucous membranes and internal organs, emaciation, enlarged lymph nodes, oedema of the skin, and sometimes death. The disease is of economic importance as it can cause a temporary reduction in milk production, temporary or permanent sterility in bulls, damage to hides and death due to secondary bacterial infections [2].

Lumpy skin disease is primarily a disease of cattle, but clinical cases have also been reported in Asian water buffalo (Bubalus bubalis). Sheep and goats seem to be unaffected even when they are in close contact with cattle during outbreaks. The situation in wild ungulates is currently unclear [3].

\section{Materials and Methods}

Materials for LSDV antigen detection have been collected at postmortem from skin nodules, and lymph nodes of suspected being infected cattle. The diagnosis was performed by real-time PCR method based on protocol described in OIE Terrestrial Manual [2].

The live attenuated sheep pox vaccine was used (produces from RF) where sheep pox virus was cultivated on the tissues of testicles of lambs or in horioallantoisa of chicken embryos. The cattle above 6 months were vaccinated once hypodermically by $x 10$ doze of vaccine.

\section{Results}

Up to 2015 the LSD was never registered in Armenia and first clinical cases of LSD was seen in southern part of Armenia (near border with Iran) in the end of 2015 among cattle which were kept in pastures. The samples which were taken from dead animals were positive for LSD in RT-PCR and additional diagnosis was done in Federal Governmental Budgetary Institution "Federal Centre for Animal Health" (Vladimir, Russian Federation) which confirmed the presence of LSDV.

More likely the LSDV was introduced in country from Iran or Turkey because only this two countries among neighboring countries previously had announced about LSD cases among livestock. The source of infection and way of transmission are not identi ied but it is known that LSDV is thought to be transmitted primarily by arthropod vectors, such as Mosquitoes, biting lies (e.g., Stomoxys calcitrans, Biomyia fasciata), Culicoides midges and hard ticks (e.g., Amblyomma hebraeum, Rhipicephalus spp.) are currently thought to be mechanical vectors. Ticks probably play little or no role when LSDV is spreading rapidly during epizootics; however, they might be involved in transmission and maintenance in endemic regions. Prolonged virus survival has been reported in some vectors. Experimentally infected Aedes aegypti were infectious for 6 days, and transovarial and transstadial transmission were demonstrated in some species of ticks. Some lying arthropods, such as Culicoides, might introduce LSDV to new areas when they are carried by the wind $[4,5]$.

Based on our investigations among above mentioned type of vectors which are able to transmit LSDV in Armenia are existing Mosquitoes, Stomoxys type of lies and Rhipicephalus spp. ticks which can be possible mechanical sources for disease transmission but not for long distances and only certain period of time when there are appropriate climate conditions. Anyway the irst case was registered during December in southern part of country and the temperature in this period of year is not enough for presence of above mentioned insects, so more likely disease was introduced through infected live animals or infected feed or other materials (Figures 1 and 2).

Since the LSD was registered in country first time and all cattle were intact for LSDV the infections among cattle were occurred with strong clinical signs and with mortality cases. In order to control further spreading of disease and control the situation was decided along with overall veterinary-sanitary measures (for example quarantine in 
Citation: $\quad$ Markosyan T, Sargsyan K, Kharatyan S, Elbakyan H, Hakobyan V, et al. (2017) Lumpy Skin Disease in Armenia. J Vet Sci Technol 8:

Page 2 of 2

infected communities, culling of infected animals, disinfections and etc.) to conduct also vaccination campaign in high risk zones based on epidemiological situation inside the country and in neighboring countries.

Was taken account that only live attenuated vaccines against LSD are currently commercially available. Because of antigenic homology and cross-protection between sheep pox, goat pox and LSD viruses, any of these viruses can be used as a vaccine strain to protect cattle against LSD [4].

In OIE Terrestrial Manual described that all strains of Capripoxvirus examined so far, whether of bovine, ovine or caprine origin, share a major neutralizing site, so that animals recovered from infection with one strain are resistant to infection with any other strain. Consequently, it is possible to protect cattle against LSD using strains of Capripoxvirus derived from sheep or goats. In 1989 and 1990 the Romanian strain of sheep pox vaccine was used to help control the LSD outbreak in Egypt. However, it is essential to carry out controlled trials, particularly using the most susceptible breeds in peak lactation, prior to introducing a vaccine strain not usually used in cattle. The duration of protection provided by LSD vaccination is unknown.

During 2016 in total 344312 cattle have been vaccinated which is $49 \%$ from total population of cattle in country (701 535 cattle). In Syunik region where was registering LSD cases during 2016 were vaccinated 95233 cattle which is $27.6 \%$ from total number of vaccination (344312) in all 10 regions of Armenia.

During last 9 months of 2017 already 143206 cattle have been vaccinated and $43.2 \%$ (61961 cattle) of them only in Syunik region. The total number of vaccinated cattle in 2017 expected to be less than 2016 because of during last year have not been registered any new cases of LSD in other regions of country and main part of target vaccination concentrated in Syunik region as a only area where was occurred LSD.

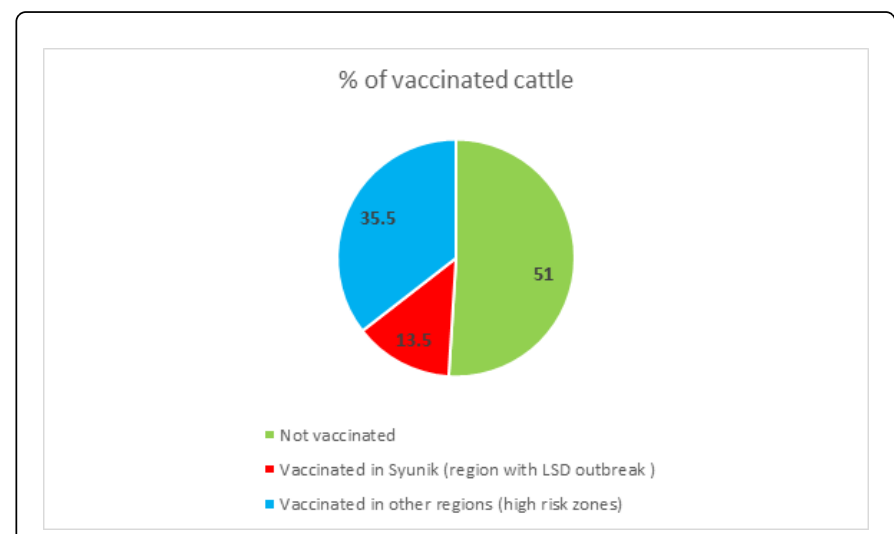

Figure 1: Proportion of vaccinated with LSD cattle (data from 2016).

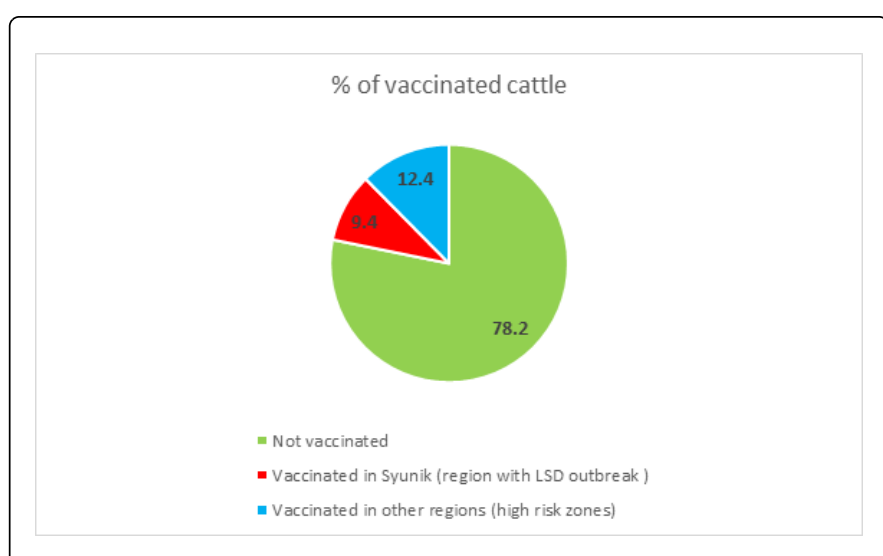

Figure 2: Proportion of vaccinated with LSD cattle (data from 2017 January-September).

\section{Conclusion}

- LSDV was registered in southern part of Armenia in December of 2015 most likely from Iran or Turkey which are neighboring countries and had announced about presence of LSD previously.

- The role of vectors in LSD epidemiology in Armenia is not clear but there are some insects presented in country and which are known as a vector of LSDV transmission.

- The use of sheep pox virus vaccine in LSD high risk zones support to prevent further spreading of disease and can be considering as an emergency tool for prevention and control of LSDV spreading.

\section{References}

1. Buller RM, Arif BM, Black DN, Dumbell KR, Esposito JJ, et al. (2005) Poxviridae. In: Fauquet CM, Mayo MA, Maniloff J, Desselberger U, Ball LA (eds) Virus Taxonomy: Eight Report of the International Committee on the Taxonomy of Viruses. Elsevier Academic Press, Oxford, pp: 117-133.

2. OIE Terrestrial Manual (2016) Chapter 2.4.13. Lumpy Skin Disease.

3. Khalafalla AI (2017) Lumpy Skin Disease (Knopvelsiekte, PseudoUrticaria, Neethling Virus Disease, Exanthema Nodularis Bovis): Emerging Infectious Diseases in Camelids, Springer, pp: 309-326.

4. Kitching RP (1983) Progress towards sheep and goat pox vaccines. Vaccine 1: 4-9.

5. Tuppurainen ES, Oura CA (2011) Review: Lumpy skin disease: An emerging threat to Europe, the Middle East and Asia. Transbound Emerg Dis 59: 40-48. 